Check for updates

Cite this: RSC Adv., 2020, 10, 3837

\title{
Characterization of the defect structure of oxide nanoparticles with the use of deuterium probes
}

\begin{abstract}
Vladimir B. Vykhodets (D) and Tatiana E. Kurennykh
The method of using deuterium probes was proposed for studying the defect structure of oxide nanoparticles. It was based on the fact that in the course of annealing nanoparticles in deuterium, clusters consisting of point defects and deuterium atoms are formed. The content of the clusters depended on the type of defects. The concentration of deuterium and the content of the clusters were determined by the method of nuclear reactions. The technique was applied to study the defect structures of the nanoparticles of YSZ10, cubic zirconium dioxide doped with yttrium, and $\mathrm{TiO}_{2}$. The nanopowders of $\mathrm{YSZ10}$ and $\mathrm{TiO}_{2}$ were synthesized by means of the laser evaporation of the ceramic target; besides, $\mathrm{TiO}_{2}$ was obtained using the sol-gel method. It was shown that the method of the use of deuterium probes allowed one to reveal the presence of one or several types of point defects in nanoparticles and determine their concentration in the bulk and near the surface of nanoparticles with an accuracy of several percent and sensitivity of about 0.01 at $\%$.
\end{abstract}

Received 16th July 2019

Accepted 11th November 2019

DOI: 10.1039/c9ra05451k

rsc.li/rsc-advances

these methods in the case of nanopowders compared to that of single crystals because of the effects of size factors as well as the practical impossibility of preparing clean crystallographically oriented sample surfaces as in the case of single crystals. In this context, the development and employment of techniques aimed at the investigation of the defect structure of nano-sized objects is a topical problem.

In the current work, for this purpose, we suggest the method of the use of deuterium probes. It is based on the hypothesis of the unambiguous relation of the concentration of the point defects in a nanoparticle $\left(C_{d}\right)$ with the deuterium concentration $\left(C_{\mathrm{D}}\right)$ in a powder subjected to annealing in gaseous deuterium. The statement of the research problem using this hypothesis looks reasonable since with the unambiguous interrelation of $C_{\mathrm{D}}$ and $C_{d}$, the method of using deuterium probes will be by far more efficient than all the techniques applied to measure the concentration of the point defects in nanoparticles before. The method of nuclear reactions (NRA) used to measure the concentration of deuterium in powders possesses high metrological characteristics; by using this method, the concentration $C_{\mathrm{D}}$ can be measured with an accuracy of several percent, and its sensitivity is at a level of $10^{-2}$ at $\%$. Accordingly, this gives grounds to assume that the method of using deuterium probes would allow the determination of the defect concentration at the same level. At the same time, because of the absence of data on the influence of point defects on $C_{\mathrm{D}}$ in oxides, we cannot rely on this method for identifying types of defects. This challenge should be addressed with the use of other methods. The choice of deuterium for probe testing rather than the lightest isotope of hydrogen, i.e., protium is explained by the fact that the
M. N. Mikheev Institute of Metal Physics, Ural Branch of the Russian Academy of Sciences, 18 S. Kovalevskaya St., Yekaterinburg, 620137, Russia. E-mail: vykhod@ imp.uran.ru 
concentration of protium cannot be determined with high accuracy and sensitivity. It is compulsory for the realization of the method of using deuterium probes that upon annealing powders in deuterium, the defect structure of the nanoparticles must not change and in the defect-free nanoparticles, deuterium virtually does not dissolve. These conditions do not look exotic since in solids, deuterium rapidly diffuses even at low temperatures, whereas in some defect-free oxides, the solubility of hydrogen is close to zero. At least, this is the case for $\mathrm{TiO}_{2}{ }^{13}$

\section{Experimental}

The main object under study was the nanopowders of zirconium dioxide YSZ10 doped with yttrium. This material is widely used in engineering. The content of yttrium oxide was made up $10 \mathrm{~mol} \%$; the chemical formula is $\mathrm{Zr}_{0.82} \mathrm{Y}_{0.18} \mathrm{O}_{1.91}$. These powders were synthesized by the laser evaporation of ceramic target; the technology and powder characterization are described in ref. 14 . The specific areas $(S)$ of nanopowders were $41,83,171$, and $243 \mathrm{~m}^{2} \mathrm{~g}^{-1}$. The choice of YSZ10 was substantiated by an idea to treat a general case of applying the new technique to nanoparticles that contain both point defects arising in the course of synthesis and the defects the existence of which in the oxide is controlled by electrical neutrality of the oxide. YSZ10 is referred to such oxides since cations of zirconium $\mathrm{Zr}^{4+}$ and yttrium $\mathrm{Y}^{3+}$ have different charge, which causes the presence of oxygen vacancies in the crystal lattice of the oxide. The concentration of thermodynamically equilibrium oxygen vacancies in the oxide $\mathrm{Zr}_{0.82} \mathrm{Y}_{0.18} \mathrm{O}_{1.91}$ was 3 at\%. Here from, in the text, the concentrations of vacancies, oxygen, deuterium, and point defects are given in percent of the total number of atoms in the oxide.

To gain information on the applicability of the method of deuterium probes for other technologies of synthesizing nanopowders and oxides of other chemical compositions, the investigation of the defect structure was performed on the nanoparticles of $\mathrm{TiO}_{2}$ in the powders synthesized by both the sol-gel method and laser evaporation of ceramic target. In the first case, the powder had the structure of anatase with a specific surface area of $50 \mathrm{~m}^{2} \mathrm{~g}^{-1}$. In the second case, the powder contained phases of both anatase and rutile with the specific surface area of $218 \mathrm{~m}^{2} \mathrm{~g}^{-1}$. The techniques of synthesis and characterization of powders are described in ref. 13.

The concentration $C_{\mathrm{D}}$ was determined on the as-synthesized nanopowders after annealing in deuterium with the help of NRA using a 2 MB Van-de-Graff accelerator and reaction ${ }^{2} \mathrm{H}(\mathrm{d}$, p) ${ }^{3} \mathrm{H}$, the energy of the primary beam being $650 \mathrm{keV}$. Most measurements on the accelerator were performed at room temperature of the specimen. In several cases, the temperatures of the powder in the NRA measurements were -70 and $60{ }^{\circ} \mathrm{C}$, and the sample holder described in ref. 15 was used. For the experiments, the particles of the nanopowders were pressed into an indium plate. The diameter of the incident beam of deuterons was $2 \mathrm{~mm}$. The products of the nuclear reaction were registered with the use of a silicon surface-barrier detector, the angle of registration being $160^{\circ}$. The irradiation dose was determined by using a secondary monitor with a statistic error of about $1 \%$. To determine the $C_{\mathrm{D}}$ values from the products of the nuclear reactions, the spectra of the samples under study were compared with that of the reference with a constant-in-depth concentration of deuterium by the procedure described in ref. 13-15, the reference being $\mathrm{ZrCr}_{2} \mathrm{D}_{0.12}$. The mean-square error in the measurements of $C_{\mathrm{D}}$ was $\sim 5 \%$. Upon irradiation, implantation of the deuterium ions into samples occurred. The increase in the deuterium concentration caused by implantation was not essential though, even at very low concentrations $C_{\mathrm{D}}$.

Annealing of the powders in deuterium, oxygen, and argon was performed in a quartz tube. The temperature gradient in the zone of the sample location was virtually absent. To measure temperature, chromel-alumel thermocouple element was used, the accuracy being $1^{\circ} \mathrm{C}$. The oxygen pressure was $0.3 \mathrm{~atm}$ and that of argon was $1 \mathrm{~atm}$. To determine the optimal conditions for annealing powders, preliminary experiments were carried out. It was established that the concentration $C_{\mathrm{D}}$ decreased if prior to annealing in deuterium, the powders were annealed in oxygen. Moreover, if the temperature of annealing was rather high, the concentration $C_{\mathrm{D}}$ was practically certain zero. For YSZ10, such temperatures were above $400{ }^{\circ} \mathrm{C}$, whereas for $\mathrm{TiO}_{2}$, they were above $800{ }^{\circ} \mathrm{C}$. Thus, the registration of values $C_{\mathrm{D}}>0$ testified to the presence in the nanoparticles of YSZ10 and $\mathrm{TiO}_{2}$ of point defects, whose concentrations decreased after the annealing of powders in oxygen. Since the diffusion rate of oxygen in the oxide YSZ10 was several orders higher than that in $\mathrm{TiO}_{2},{ }^{\mathbf{1 8 , 1 9}}$ the value of $C_{\mathrm{D}}$ $\approx 0$ for YSZ10 was obtained at an essentially lower temperature than that for $\mathrm{TiO}_{2}$. Also, these experiments indicated that the method of deuterium probes was insensitive to structure oxygen vacancies that are present in the oxide YSZ10, which is thermodynamically conditioned. Besides, the preliminary experiments showed that upon annealing of the powders in deuterium at temperatures $T<400{ }^{\circ} \mathrm{C}$, the concentration $C_{\mathrm{D}}$ increased with time of annealing $t$, which purports that at low temperatures there cannot be unambiguous correspondence between $C_{\mathrm{D}}$ and the defect structure of nanoparticles; however, at temperatures $T \geq 400{ }^{\circ} \mathrm{C}$, this did take place. In this case, the concentration $C_{\mathrm{D}}$ was higher than that in the low-temperature experiments and did not depend on $t$ upon its variation from $15 \mathrm{~min}$ to $2 \mathrm{~h}$. Such a dependence $C_{\mathrm{D}}(T, t)$ was conditioned by the fact that at $T<400{ }^{\circ} \mathrm{C}$, the limiting stage upon deuterium incoming into oxides was the dissociation of deuterium molecules at the surface of the nanoparticles, whereas at $T \geq 400{ }^{\circ} \mathrm{C}$, the rate of deuterium diffusion was high enough to provide homogeneous and thermodynamically equilibrium distribution of deuterium in nanoparticles. Taking into account the results of preliminary experiments, the annealing in deuterium was carried out at $400{ }^{\circ} \mathrm{C}$. To elevate the temperature was unreasonable since it could result in changes in the defect structure of the nanoparticles. The duration of annealing in deuterium was $1 \mathrm{~h}$ and the deuterium pressure was $0.6 \mathrm{~atm}$. Changing the pressure upon annealing over a wide range as well as its duration, did not affect $C_{\mathrm{D}}$. 


\section{Results and discussion}

\subsection{Nanopowders YSZ10}

The inset in Fig. 1 shows two spectra of the products of the ${ }^{2} \mathrm{H}$ $(\mathrm{d}, \mathrm{p})^{3} \mathrm{H}$ reaction. They correspond to the samples with a constant-in-depth deuterium concentration. For mathematically processing, an output of the ${ }^{2} \mathrm{H}(\mathrm{d}, \mathrm{p})^{3} \mathrm{H}$ reaction in a fixed energy range that corresponds to the oxide thickness of about $2.7 \mu \mathrm{m}$ was used. We investigated the deuterium concentration $\left(C_{\mathrm{D}}\right)$ depending on the dose of deuteron irradiation $(N)$. Fig. 1 displays that at low irradiation doses, the concentration $\left(C_{\mathrm{D}}\right)$ decreases on increasing $N$, while at large $N$, it does not depend on the irradiation dose. This dependence was observed for both the as-synthesized nanopowders and those subjected to oxygen annealing. The effect of the decrease in $C_{\mathrm{D}}$ upon deuteron irradiation did not depend on the temperature of the nanopowders.

The data on $C_{\mathrm{D}}(N)$ give grounds to suggest that deuterium atoms form weak and strong chemical bonds with point defects. The weak bonds broke under deuteron irradiation, while the strong ones remained intact. When the weak bonds broke, free deuterium atoms were formed; they could leave nanoparticles or be captured by other point defects. These mechanisms were competing. Let us designate the concentrations of deuterium with weak and strong bonds in powders prior to the deuteron irradiation as $C_{1}$ and $C_{2}$, respectively. For all the as-synthesized powders, within the experimental error, we obtained the integer ratio of the deuterium concentrations with weak and strong bonds: $C_{1} / C_{2}=2.0 \pm 0.1$. These results indicate that the annealing of the as-synthesized nanopowders in deuterium resulted in the formation of the clusters of the composition $d \mathrm{D}_{m}^{(1)} \mathrm{D}_{n}^{(2)}$ ( $d$ is the point defect), which arose upon the synthesis of the nanopowder; $m$ and $n=1,2,3 \ldots$; the upper indexes (1)

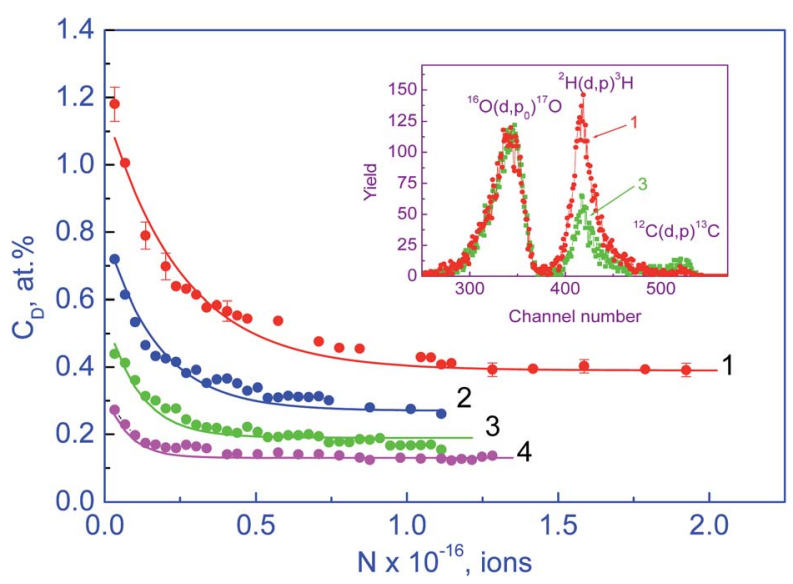

Fig. 1 Dependences of deuterium concentration $C_{D}$ on deuteron irradiation dose $N$ for YSZ10 powders. Curves 1, 2, 3 - for the assynthesized powders with the specific areas $S=171,83$, and $41 \mathrm{~m}^{2} \mathrm{~g}^{-1}$, respectively; curve $4-$ for the powder annealed in oxygen at $350^{\circ} \mathrm{C}$ for $15 \mathrm{~min}$ : points - experimental data, lines - calculation according to (2) with $\lambda=2$. The inset presents the spectra of the nuclear reaction products for nanopowders with $S=41 \mathrm{~m}^{2} \mathrm{~g}^{-1}$ (curve 3) and $171 \mathrm{~m}^{2} \mathrm{~g}^{-1}$ (curve 1). and (2) designate the deuterium atoms D weakly and strongly bound with the defects and the lower indexes $m$ and $n$ designate the number of deuterium atoms in a cluster. For the clusters in the as-synthesized powders of YSZ10, the condition $m=2 n$ was met.

To determine the values $m$ and $n$, the experimental data on $C_{\mathrm{D}}(N)$ from the range where $C_{\mathrm{D}}$ decreases on increasing $N$ were used. Let us assume that the deuterium atoms that are formed upon breaking weak bonds leave nanoparticles for vacuum. This assumption corresponds to a low concentration of point defects and a small size of nanoparticles. In this model, the dependence $C_{\mathrm{D}}(N)$ can be described with the differential equation

$$
\mathrm{d} C_{\mathrm{D}}=-\frac{\lambda C_{\mathrm{D}}}{N_{0}} \mathrm{~d} N
$$

where $N_{0}$ is the number of deuterium atoms in the irradiated volume with weak bonds at the starting instant; $\lambda$ is the coefficient of breaking of weak bonds, which is equal to the amount of weak bonds broken by one deuteron. From eqn (1), it is easy to obtain

$$
C_{\mathrm{D}}=C_{1} \exp \left(-\frac{\lambda N}{N_{0}}\right)+C_{2}
$$

As is seen from Fig. 1, the experimental data on $C_{\mathrm{D}}(N)$ are satisfactorily described by expression (2) at $\lambda=2$; hence, clusters consisting of defects and deuterium atoms had the composition $d \mathrm{D}_{2}^{(1)} \mathrm{D}_{1}^{(2)}$. With this in mind, we obtained the expression for the concentration of point defects $C_{d}$, which were formed upon the synthesis of the YSZ10 nanopowders:

$$
C_{d}=\frac{1}{3} C_{\mathrm{D}}=\frac{1}{2} C_{1}=C_{2}
$$

The results on the concentration of defects $C_{d}$ in the assynthesized powders that were obtained with expression (3) are given in Fig. 2 (straight line 1). The values of $C_{d}$ range from
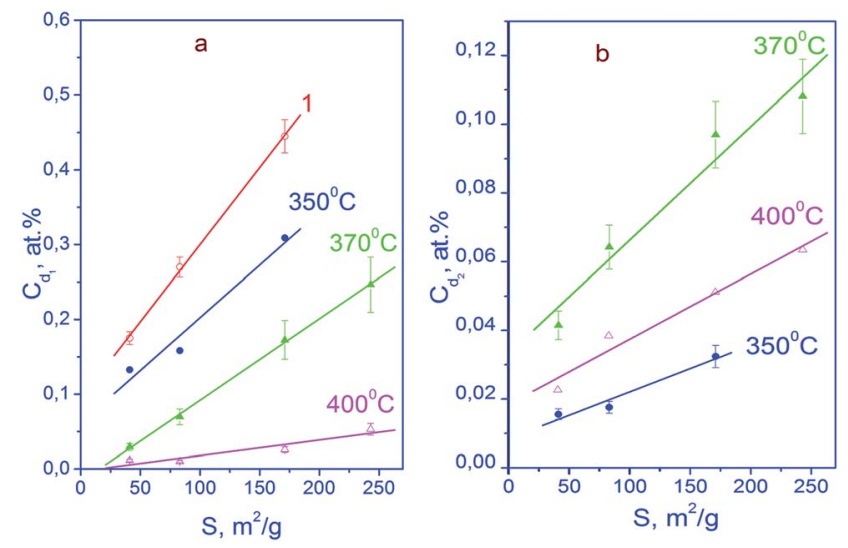

Fig. 2 Dependences of concentrations of point defects $C_{d 1}(a)$ and $C_{d 2}$ (b) on the specific surface area $S$ of nanopowders of the YSZ10 oxide: 1 - results for as-synthesized powders obtained using eqn (3), the others, for the powders subjected to annealing in oxygen for $15 \mathrm{~min}$, using eqn (5). Annealing temperatures are shown in the panels. 
$\sim 0.18$ to $\sim 0.45 \%$; the observed dependence $C_{d}(S)$ is linear. Evidently, the defects that are registered with the method of deuterium probes are not structure vacancies in the oxygen sublattice. As noted in section 2, the concentration of the structure vacancies in the oxygen sublattice in the oxide YSZ10 amounts to $3 \%$ and does not depend on the specific area $(S)$ of the powder. It is pertinent to make a remark that the technique of using deuterium probes as well as others say EPR is by no means sensitive to any type of point defects.

For many applications, it is of interest to gain data together with the total concentration of defects $C_{d}$ on their concentration in the bulk $C_{\mathrm{dv}}$ and near the surface $C_{\mathrm{ds}}$ of nanoparticles. They can also be determined using deuterium probes. To specify a spherical form of nanoparticles, eqn (3) can be easily transformed to

$$
C_{d}=\frac{M}{9 N_{A}}\left(\frac{\omega}{\rho}+\sigma S\right) \text { or } C_{d}=C_{\mathrm{dV}}+\left(C_{\mathrm{dS}}-C_{\mathrm{dV}}\right) \Delta \rho S
$$

where $M$ and $\rho$ are the molar mass and density of oxides; $N_{\mathrm{A}}$ is the Avogadro number, $\omega$ and $\sigma$ are the densities of deuterium atoms in the bulk and near the surface of nanoparticles, and $\Delta$ is the thickness of the surface atomic layer in the oxide particles, which can be assumed to be $\Delta \approx 0.5 \mathrm{~nm}$. It can be inferred from eqn (4) that in such a model, $C_{d}(S)$ is a linear function if the densities and concentrations, i.e., $\omega$ and $\sigma, C_{\mathrm{dv}}$ and $C_{\mathrm{ds}}$ do not depend on the specific area $(S)$ of the powder. From Fig. 2, line 1 , it is seen that the plot $C_{d}(S)$ is linear and using eqn (4), one can determine the parameters that characterize the spatial distribution of the point defects in the nanoparticles as well as their densities and concentrations in the bulk and near the surface. It was observed that the concentrations of the point defects formed upon the synthesis of the YSZ10 nanoparticles with the use of the technology of the laser evaporation of a ceramic target were $C_{\mathrm{dV}} \approx 0.1$ at\% and $C_{\mathrm{ds}} \approx 0.8$ at\%. The data on $C_{\mathrm{dv}}$ and $C_{\mathrm{ds}}$ reliably evidenced a higher concentration of point defects near the surface than in the bulk. Such quantitative information was gained for the first time and is important for the catalysis and interpretation of the magnetic properties of oxide nanopowders. In particular, the method of using deuterium probes can reasonably be used to verify the hypothesis on the existence of the so-called $\mathrm{d}^{\circ}$-magnetism. This hypothesis was used in ref. 16 to explain the ferromagnetic properties of oxide nanopowders.

To obtain information on the types and properties of point defects, which were detected via the method of using deuterium probes, we investigated the influence of the annealing of the YSZ10 powders in argon and oxygen on the deuterium concentration by means of EPR. The annealing of the assynthesized powders in argon and oxygen was performed prior to their treatment in deuterium. The annealing in highpurity argon was carried out at $400{ }^{\circ} \mathrm{C}$ for $15 \mathrm{~min}$, whereas the annealing in oxygen was conducted in the range from 350 to $400{ }^{\circ} \mathrm{C}$ for $15 \mathrm{~min}$ as well. No peaks were present in the electron paramagnetic resonance (EPR) spectra of both as-synthesized powders and those annealed in deuterium. Hence, a conclusion can be made that as evidenced by the method of using deuterium probes, the defects in the YSZ10 nanoparticles are not paramagnetic centers. As mentioned above, they are no structure vacancies in the oxygen sublattice of oxides as well; the question on the type of these defects needs further investigations.

The annealing of the powders in argon at $400{ }^{\circ} \mathrm{C}$ did not result in a noticeable change in the deuterium concentrations $C_{\mathrm{D}}, C_{1}$, and $C_{2}$. This indicated either the immobility of the defects registered by the method of using deuterium probes at $400{ }^{\circ} \mathrm{C}$ or the absence of interactions between them. Contrary to this, the annealing in oxygen led to a significant decrease in the deuterium concentrations. In this relation, we suppose that the defects that are present in the YSZ10 nanoparticles and registered by the method of deuterium probes occur only due to the oxygen deficit in the nanoparticles, which can arise upon the synthesis of the powders. ${ }^{\mathbf{1 4}}$ On increasing the temperature of annealing in oxygen, the deuterium concentrations $C_{\mathrm{D}}, C_{1}$ and $C_{2}$ monotonously decreased and after annealing at $400{ }^{\circ} \mathrm{C}$, they were an order of magnitude lower than that for the assynthesized powders. These results were obviously conditioned by the decrease in the concentration of point defects after annealing in oxygen. The ratios of concentrations $C_{1} / C_{2}$ also monotonously decreased on increasing the temperature of annealing and fell in the range from 2 to 0.5 . Moreover, the ratios $C_{1} / C_{2}$ for the annealed powders, unlike those for the starting ones, were not integers and indicated that after synthesis, in the nanoparticles, there dominated a single type of point defects, whereas after annealing in oxygen, there occurred point defects of other types as well. In accordance with this, eqn (3) is valid only if the defects of one type are present in the nanoparticles; otherwise, in a general case, when applying the method of using deuterium probes, it is necessary to use the expression

$$
C_{\mathrm{D}}=\sum C_{d i}\left(m_{i}+n_{i}\right)
$$

where $C_{d i}$ and $m_{i}$ and $n_{i}$ are the concentration of the defects of the $i$-th type in a nanoparticle and the numbers of deuterium atoms with a strong and weak bonding in such a cluster, respectively. In this context, we relate the point defects to different types if the compositions of the clusters consisting of deuterium atoms and defects, i.e., $m_{i}$ and $n_{i}$ are different.

The defect concentrations and the values of $m_{i}$ and $n_{i}$ can be determined from the experimental data on $C_{\mathrm{D}}, C_{1}$ and $C_{2}$ using eqn (5); however, at a large number of defects of different types, this procedure will not give unambiguous results. At the same time, for the powders of YSZ10 that were subjected to annealing in oxygen, it was observed that summation in (5) can be reduced to only two types of point defects. Accounting for a larger number led to contradictions with the experimental data on $C_{\mathrm{D}}$, $C_{1} / C_{2}, C_{1}$, or $C_{2}$. This result was obtained upon the variation in the $m_{i}$ and $n_{i}$ values over a wide range. Based on this, a conclusion was made that in the nanoparticles of YSZ10 after their annealing in oxygen, the defects of only two types are present. In Fig. 2, their concentrations are denoted as $C_{d 1}$ and $C_{d 2}$. They were determined using the experimental data on $C_{\mathrm{D}}, C_{1} / C_{2}$ and

the expression $\frac{C_{1}}{C_{2}}=\frac{2 C_{d 1}+C_{d 2}}{C_{d 1}+C_{d 2}}$ which follows from the 
characteristics of the defects designated as $i=1$ and $i=2$. The value $i=1$ in eqn (5) corresponds to the clusters for which $m_{1}=$ 3 and $n_{1}=1$. Note that the defects of this type were present in the as-synthesized powders. The value $i=2$ corresponds to the clusters for which $m_{2}=1$ and $n_{2}=0$. As seen from Fig. 2, the concentration $\left(C_{d 1}\right)$ of the defects, which were present in the assynthesized powders, decreased upon annealing in oxygen and defects of another type were formed. Their concentration $C_{d 2}$ increased after increasing the temperature of annealing from 350 to $370{ }^{\circ} \mathrm{C}$ and decreased with the further increase in the temperature to $400{ }^{\circ} \mathrm{C}$. The summed concentration $C_{d 1}+C_{d 2}$ of the point defects decreased in the course of annealing by approximately 5 times, whereas the ratio of concentrations $C_{d 1} /$ $C_{d 2}$ monotonously decreased from $\sim 10$ to $\sim 0.7$ on increasing the temperature of annealing. The procedure of the determination of the defects of different types via eqn (5) is tedious and in a general case does not provide unambiguous results. At the same time, the analysis showed that to gain approximate estimates, eqn (3) can be used; the error in the determination of the summed concentration of defects is no more than $20 \%$. Such accuracy is acceptable in many cases.

Thus, via the method of using deuterium probes, it was established that in the nanoparticles of YSZ10 synthesized by the laser evaporation of the ceramic target, point defects are present, whose concentration in the bulk of the nanoparticles is about $0.1 \%$, whereas in the subsurface layers, it is higher by an order of magnitude. The types of defects over the body of nanoparticles do not differ and are conditioned by the oxygen deficit in nanoparticles. The concentration of the defects does not change after annealing in argon but decreases after annealing in oxygen. Also, it is established that the detected defects are neither oxygen vacancies nor paramagnetic centers.

\subsection{Nanopowders of $\mathrm{TiO}_{2}$}

It is seen from Fig. 1 and 3 that the dependences of the deuterium concentration on the dose of deuteron irradiation $C_{\mathrm{D}}(N)$ for the as-synthesized powders of $\mathrm{YSZ10}_{0}$ and $\mathrm{TiO}_{2}$ produced by two different technologies are similar: at small doses, the concentration $C_{\mathrm{D}}$ decreases on increasing $N$ and at high $N$, it does not depend on the irradiation dose. Besides, in all cases, the ratio of the concentrations of deuterium atoms with weak and strong bonding, i.e., $C_{1} / C_{2}$ is an integer. However, the very quantities $C_{1} / C_{2}$ are different. For the powders of YSZ10, the value is 2.0 , whereas for $\mathrm{TiO}_{2}$, it is 1.0 for both synthesis technologies. These results indicate that the compositions of the clusters in the as-synthesized powders of YSZ10 and $\mathrm{TiO}_{2}$ are different. For the oxide YSZ10, the cluster contains two deuterium atoms strongly bound with the defects and one deuterium atom with weak bonding. In the case of oxide $\mathrm{TiO}_{2}$, the cluster was composed of one deuterium atom with strong bonding and one with weak bonding. The method of using deuterium probes gave the following values of the defect concentrations $C_{d}$ in the as-synthesized $\mathrm{TiO}_{2}$ powders: $0.15 \%$ and $0.42 \%$ for the powders synthesized by the sol-gel method and the laser evaporation of the ceramic target, respectively. These values were close to the

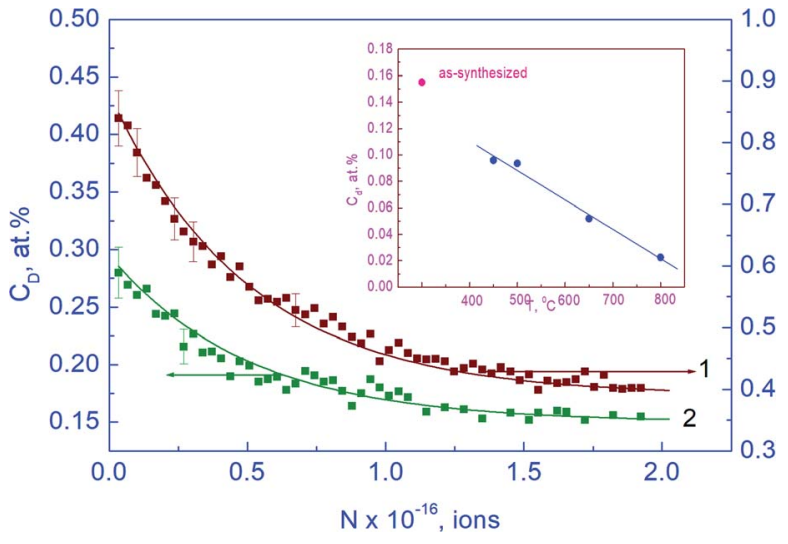

Fig. 3 Dependences of deuterium concentration $C_{D}$ on deuteron irradiation dose $N$ for $\mathrm{TiO}_{2}$ powders. Curves 1 and 2 are for the assynthesized powders obtained with technologies of laser evaporation of ceramic target and sol-gel method, respectively. The specific surface areas $S=218$ and $50 \mathrm{~m}^{2} \mathrm{~g}^{-1}$, respectively: points - experimental data, lines - calculation results according to (2) with $\lambda=1$. In the inset, the dependence of defect concentration $C_{d}$ on the temperature of oxygen annealing $T$ is shown for the powder synthesized by the sol-gel method.

concentrations of the defects $C_{d}$ in the nanopowders of YSZ10 at equal specific areas of the powder surface, i.e., the dependences $C_{d}(S)$ for the powders of YSZ10 and $\mathrm{TiO}_{2}$ differed insignificantly. This result was hardly trivial as it was obtained for the powders of different oxides synthesized by different technologies. In view of this, it will be of interest to gain data on the dependences $C_{d}(S)$ for a wide variety of oxide powders and synthesis technologies.

In the inset in Fig. 3, the temperature dependence of the defect concentration is shown for the $\mathrm{TiO}_{2}$ powders synthesized by the sol-gel method and subjected to annealing in oxygen. The duration of annealing at each temperature was $15 \mathrm{~min}$. The summed concentration of defects was obtained by the formula $C_{\mathrm{d}}=\frac{1}{2} C_{\mathrm{D}}=C_{1}=C_{2}$, which is analogous to eqn (3). Such a procedure gives approximate results and its accuracy does not exceed $20 \%$, as mentioned in section 3.1. It was seen that annealing in oxygen led to the decrease in the defect concentration. These results for the powders of $\mathrm{TiO}_{2}$ and YSZ10 were similar. EPR spectroscopy studies were performed on the assynthesized powders of $\mathrm{TiO}_{2}$ produced by both technologies as well as on the powders subjected to annealing in deuterium. The peaks in the spectra were registered only for the powders assynthesized by the sol-gel method and those subjected then to annealing in deuterium; the results are shown in Fig. 4. In accordance with the data, ${ }^{20,21}$ these peaks evidence the presence of $\mathrm{Ti}^{3+}$ ions and oxygen vacancies in the nanoparticles. However, the concentration of the paramagnetic centers turned out to be much smaller that the concentration of the defects determined by the method of using deuterium probes. The corresponding values were approximately 0.003 and $0.15 \%$. Hence, it can be stated that the methods of EPR and deuterium probes register in the nanoparticles of $\mathrm{TiO}_{2}$ of different types of point defects. The same result was obtained for the powders of YSZ10 in section 3.1. 


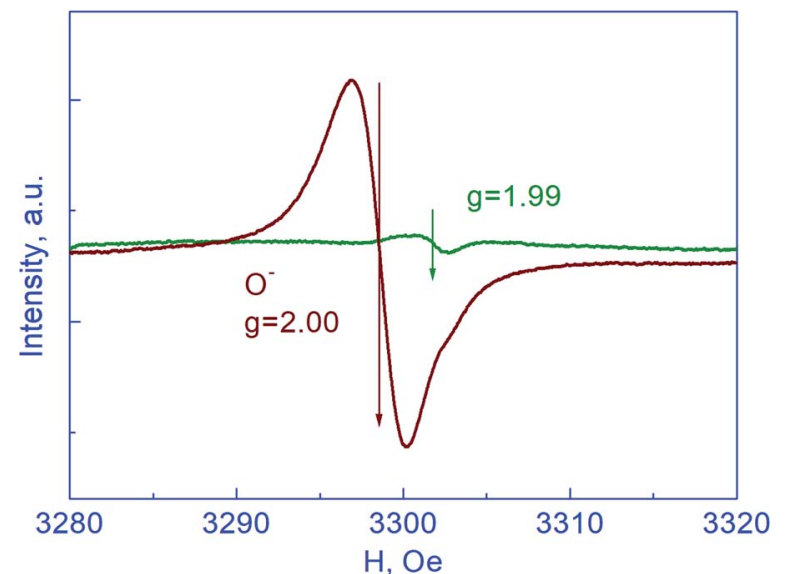

Fig. 4 EPR spectra obtained for powders of $\mathrm{TiO}_{2}$ synthesized by the sol-gel method: green line for the as-synthesized powder, wine line for that after annealing in deuterium.

It is of interest to compare the results of the defect structure of the oxide nanoparticles obtained using traditional approaches and by means of the method of using deuterium probes. For YSZ10, such information is unavailable in literature; therefore, we ran the comparison with some data on $\mathrm{TiO}_{2}$ nanoparticles. We observed a decrease in the concentration of point defects after annealing powders in oxygen, which was similar to the case of the application of positron annihilation for studying the defect structure in the $\mathrm{TiO}_{2}$ nanoparticles ${ }^{7}$ synthesized as in our work by the sol-gel method. In this work, it was also established that the prevailing type of point defects in the $\mathrm{TiO}_{2}$ nanoparticles were the $3 \mathrm{D}$ clusters of oxygen vacancies rather than monovacancies. The method of using deuterium probes could not help us identify the type of point defects either but allowed a conclusion to be made, that is, these defects are different from monovacancies in the oxygen sublattice. In the current work, we did not find any differences in the behaviors of point defects and the types of defects localized in the bulk and near the surface of nanoparticles. As already noted, the difference was found only in the concentrations of these defects. In ref. 17, by means of EPR, $\mathrm{TiO}_{2}$ nanopowders doped with iron were investigated in the as-prepared state and after etching in HCl. In the course of etching, the specific surface area of the nanopowders virtually did not change; only a thin surface layer of the nanoparticles was removed. The shape of the EPR spectra did not change after etching, while their intensity decreased approximately by a factor of 2 . These data, similar to the results of the current work, testified to the same type of defects in the bulk and near the surface of nanoparticles and to the higher concentrations of the defects near the surface. These examples show that at the qualitative level, traditional approaches and the method of using deuterium probes give analogous results; however, the new method is capable of providing quantitative information with high accuracy and sensitivity.

\section{Conclusions}

Thus, in this work, a new method for studying the defect structure of oxide nanoparticles was proposed. It included annealing nanopowders in deuterium and their subsequent irradiation with deuterons in an accelerator. When irradiated by means of NRA, it was possible to measure the concentration of deuterium in the powders and determine the content of clusters that were formed upon annealing in deuterium. The clusters consisted of point defects and deuterium atoms, which formed strong and weak bonds with the defects. The method of using deuterium probes allows one to determine the concentration of point defects near the surface and in the bulk of the oxide nanoparticles within several percent as well as to gain data on the existence in the nanoparticles of one or several types of defects. In the measurements of the defect concentrations, the sensitivity of the method was about 0.01 at $\%$. The method was approved in this work on the nanoparticles of the YSZ10 oxide and $\mathrm{TiO}_{2}$ synthesized by the laser evaporation of the ceramic target as well as on the nanoparticles of $\mathrm{TiO}_{2}$ synthesized by the sol-gel method, which gave grounds to suppose that this method can be used in the studies of the nanoparticles of other oxides produced by other technologies. The identification of the types of point defects is beyond the capacity of the abovementioned method. However, this step is possible in future if a database on the content of clusters consisting of deuterium atoms and point defects of different types in oxides can be composed. This problem requires further investigations including the traditional methods applied for the identification of the types of point defects.

\section{Conflicts of interest}

There are no conflicts of interest to declare.

\section{Acknowledgements}

The research was carried out within the state assignment of Ministry of Science and Higher Education of the Russian Federation (theme "Function" no. AAAA-A19-119012990095-0).

\section{Notes and references}

1 Z. Chen, Y. S. Zhao, J. Q. Ma, C. Liu and Y. Ma, Ceram. Int., 2017, 43, 16763.

2 J. L. S. Cuaila, W. Alayo and C. O. Avellaneda, J. Magn. Magn. Mater., 2017, 442, 212.

3 D. Li, D. K. Li, H. Z. Wu, F. Liang, W. Xie, C. W. Zou and L. X. Shao, J. Alloys Compd., 2014, 591, 80.

4 P. V. Kamat, J. Phys. Chem. C, 2012, 116, 11849.

5 S. Daothong, N. Songmee, S. Thongtem and P. Singjai, Scr. Mater., 2017, 57, 567.

6 K. Westerholt, H.-W. Becker, C. Somsen, S. Gök, A. Bashir, R. Khaibullin and L. Tagirov, J. Phys. D: Appl. Phys., 2009, 42, 115005.

7 S. Ghosh, G. G. Khan, K. Mandal, A. Samanta and P. M. G. Nambissan, J. Phys. Chem. C, 2013, 117, 8458-8467. 
8 M. Venkatesan, C. B. Fitzgerald and J. M. D. Coey, Nature, 2004, 430, 630.

9 P. Dutta, M. S. Seehra, Y. Zhang and I. Wender, J. Appl. Phys., 2008, 103, 07D104.

10 M. Stoneham, J. Phys.: Condens. Matter, 2010, 22, 074211.

11 C. Sudakara, P. Kharela, R. Suryanarayanana, J. S. Thakurc, V. M. Naikd, R. Naika and G. Lawesa, J. Magn. Magn. Mater., 2008, 320, L31.

12 N. H. Hong, J. Sakai, N. Poirot and V. Brizé, Phys. Rev. B: Condens. Matter Mater. Phys., 2006, 73, 132404.

13 V. B. Vykhodets, K. G. Johnson, T. E. Kurennykh, I. V. Beketov, O. M. Samatov, A. I. Medvedev and E. A. A. Jarvis, Surf. Sci., 2007, 665, 10.

14 V. B. Vykhodets, E. A. A. Jarvis, T. E. Kurennykh, I. V. Beketov, S. I. Obukhov, O. M. Samatov, A. I. Medvedev, A. E. Davletshin and T. Whyte, Surf. Sci., 2016, 644, 141.
15 V. B. Vykhodets, O. A. Nefedova, S. I. Obukhov, T. E. Kurennykh, S. E. Danilov and E. V. Vykhodets, JETP Lett., 2018, 107, 211.

16 G. Bouzerar and T. Ziman, Phys. Rev. Lett., 2006, 96, 207602. 17 A. Ye. Yermakov, A. F. Gubkin, A. V. Korolev, L. S. Molochnikov, M. A. Uimin, E. V. Rosenfeld, M. I. Kurkin, A. S. Minin, A. S. Volegov, D. W. Boukhvalov and S. F. Konev, J. Phys. Chem. C, 2019, 123, 1494.

18 H. Solmon, C. J. A. Monty, M. Filal, G. Petot-Ervas and C. Petot, Solid State Phenom., 1995, 41, 103.

19 D. J. Derry, D. G. Lees and J. M. Galvert, J. Phys. Chem. Solids, 1981, 42, 57.

$20 \mathrm{Yu}$. Chen, W. Li, J. Wang, Y. Gan, L. Liu and M. Ju, Appl. Catal., B, 2016, 191, 94.

21 G. Calatayud, T. Jardiel, M. Peiteado, F. Illas, E. Giamello, F. J. Palomares, D. Fernández-Hevia and A. C. Caballero, J. Phys. Chem. C, 2015, 119, 21243. 